of iodide provides evidence of hypoperfusion in chronic rheumatoid synovitis. Arthritis Rheum 1985;28:1096-104. 9 Sahn SA, Kaplan RL, Maulitz RM, Good JT. Rheumatoid pleurisy. Observations on the development of low pleural pleurisy. Observations on the development of low pleural fluid $\mathrm{pH}$.
10 Taryle DA, Good JT, Sahn SA. Acid generation by pleural fluid: possible role in the determination of pleural fluid pH. $f$ Lab Clin Med 1979;93:1041-6.

pH. F Lab Clin Med 1979;93:1041-6.
11 Pettersson T, Ojala K, Weber TH. Diagnostic significance of pleural fluid lactate concentrations. Infection 1985;13: of pleural

\title{
Epidemic outbreak of interstitial lung disease in aerographics textile workers - the "Ardystil syndrome": a first year follow up
}

\author{
Amparo Solé, Pedro J Cordero, Pilar Morales, Manuela E Martínez, Francisco Vera, \\ Carmen Moya
}

Service of Pneumology A Solé

P J Cordero

$P$ Morales

M E Martínez

Service of Pathology

F Vera

La Fe University Hospital, Valencia, Spain

Conselleria de Sanitat I Consum, Generalitat Valenciana, Valencia, Spain

C Moya Reprint requests to:
Dra Amparo Solé Jover, Bélgica 26, $40^{\mathrm{a}}$ Belgica $26,40^{\mathrm{a}}$,
Valencia 46021 , Spain. Valencia 46021, Spain. Returned to authors 19 April 1995 Revised version received 26 May 1995 Accepted for publication 30 August 1995

\begin{abstract}
Background - The longer term respiratory effects of massive inhalational exposure of textile printing sprayers to Acramin (the "Ardystil syndrome") are not well established.
\end{abstract}

Methods - A 12 month follow up of 27 heavily exposed textile sprayers was performed.

Results - Twenty one patients experienced cough, 18 dyspnoea, and 17 nose bleeding at initial exposure, with histological evidence of organising pneumonia in 13 cases, radiological abnormalities detected by computed tomographic scanning in 20 cases, and diminution of diffusion capacity to below $80 \%$ of predicted in seven cases. At one year after exposure symptoms persisted in 15 cases, radiological alterations in six, and diffusion capacity was reduced in nine.

Conclusions - Whilst most of our patients showed improvement at one year, evidence of persistent lung involvement was present in an appreciable minority of exposed cases.

(Thorax 1996;51:94-95)

Keywords: occupational lung disease, interstitial lung disease.

An epidemic outbreak of interstitial lung disease occurred in textile print spraying companies in the Autonomous Community of Valencia, Spain in $1992 . .^{12}$ During that year six workers died of a restrictive pulmonary insufficiency due to organising pneumonia associated sometimes with pulmonary fibrosis, and another worker proceeded to double lung transplantation. This has been called locally the "Ardystil syndrome", after the name of the firm in which the first deaths occurred. Investigations carried out by the Public Health Department established that the lung disease was caused by spraying procedures delivering a respirable aerosol of Acramin FWIJ (a sub- stance obtained from the reaction between a diethylenetriamine and adipic acid). ${ }^{2}$ Organising pneumonia is the main pathological feature, but the pathology ranges from nonspecific (or minor) lung lesions to pulmonary fibrosis.

In the present study we report the clinical, radiological, and functional courses of patients with this syndrome followed up over a one year period.

\section{Methods}

Approximately 90 workers in the Autonomous Community of Valencia are thought to have been affected by exposure, of whom 27 (21 women), including the most severe clinical cases, were treated in our hospital. The mean (SD) age of the patients was 23 (8) years (range 17-52), and 21 were smokers. Eight of these patients were seen specifically for assessment for possible lung transplantation. Diagnosis was confirmed by transbronchial biopsy in 24 cases, videothoracoscopy in one, and at necropsy in another. In one patient the severity of the disease prevented the performance of a pulmonary biopsy. Lung abnormalities were interpreted as minor changes (increased numbers of macrophages) in six cases, intra-alveolar knots of fibrin in eight, and organising pneumonia in 13. All 27 patients were treated with oral corticosteroids.

\section{Results}

The most frequently reported symptoms at presentation were cough (21 cases), shortness of breath (18 cases), and nose bleeding (17 cases). After one year these symptoms had significantly improved in the 26 survivors with nose bleeding persisting in one patient, cough in eight, and shortness of breath in four. In addition to the respiratory symptoms, 14 patients experienced abdominal pain, diarrhoea or nausea. Cognitive disorders (memory loss) and minor neurological manifestations (head- 

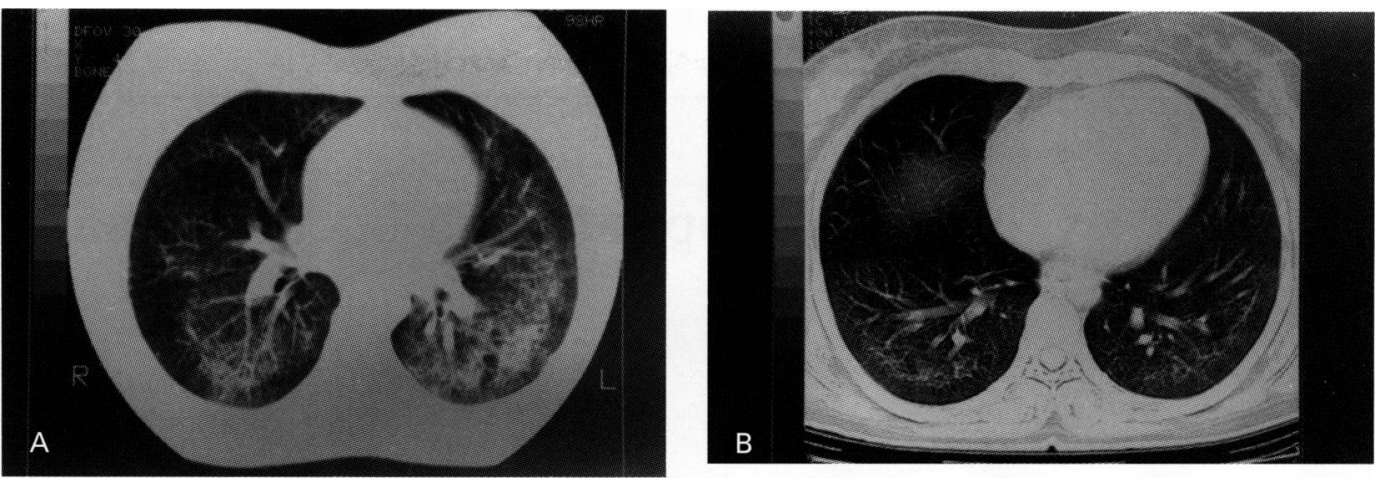

Computed tomographic (CT) scan from a patient with the Ardystil syndrome $(A)$ at diagnosis and (B) at one year. The initial CT scan shows bilateral alveolar patchy opacities. After corticosteroid treatment the CT scan shows bilateral reticular opacities.

ache, paraesthesias, and tremors) were detected in 18 patients between seven months and one year after diagnosis of the lung disease.

Computed tomographic findings at the onset of the clinical manifestations were reticulonodular opacities (eight cases), bilateral patchy infiltrates (six cases), both patchy infiltrates and reticulonodular opacities (five cases), and linear opacities (one case). After one year thoracic high resolution computed tomographic (HRCT) scanning proved normal in 20 cases $(p<0.0001)$ and linear opacities and/or reticulonodular images persisted in six patients (figure), all of whom had previously been diagnosed as having organising pneumonia.

Respiratory function testing revealed some degree of functional abnormalities in 17 patients in the course of the study, most of whom had organising pneumonia. Obstructive ventilatory defects were detected only in smokers. At the beginning of the period of follow up diffusion capacity was diminished to less than $80 \%$ of predicted in seven patients, in five of whom it constituted the only functional alteration observed. After one year diffusion was abnormal in nine patients, in four of whom it manifested for the first time. Hypoxaemia was only detected in the two patients with severe restriction.

\section{Discussion}

The so-called "Ardystil syndrome" constitutes an interstitial lung disease caused by the inhalation of toxic vapours and/or substances in aerographics textile industries that fail to comply with European Community labour health and hygiene regulations. ${ }^{2}$

The pathogenic mechanism involved in the lung disease is unknown, and the nature of the toxic agent most directly involved is not clear. Clinical data suggest that the mechanism of the lung damage is direct toxicity, with little evidence of systemic inflammation - in terms of an acute phase response - in peripheral blood or extrapulmonary abnormalities at necropsy.

The symptoms observed in our patients both at the onset of the syndrome and after one year exhibit clear differences with respect to other series of organising pneumonia described in the literature..$^{3-5}$ Thus, 17 patients presented with nose bleeding, which points to a local irritant action on the upper airways. In addition, we observed features specific to this syndrome such as the digestive disorders at the onset of the disease and the neurological impairments detected after about one year. These manifestations may be due to the recognised systemic effects of the substances involved (including naphthas, trichloroethylene, and trichloroethane) ${ }^{6}$ and/or to still unknown mechanisms.

It is well known that many patients with organising pneumonia respond well to treatment with corticosteroids, but that pulmonary fibrosis can occur despite steroid treatment. ${ }^{3}$ It has been suggested that linear opacities at the bases may be associated with a poor prognosis. ${ }^{4}$ In our series six patients with organising pneumonia appeared to evolve towards interstitial fibrosis, as reflected by the thoracic HRCT findings and respiratory function tests after one year. These patients consisted mainly of those who initially exhibited organising pneumonia with reticulonodular opacities. The patients with minor pathological changes at the first transbronchial biopsy showed complete resolution, as evidenced by functional exploration and HRCT scanning. In the patient who died the lung examination at necropsy showed characteristic features of organising pneumonia.

Our study has shown that most of our cases, who we think included some of the most severely affected individuals with Ardystil syndrome in our hospital, recovered without long term sequelae. However, a few cases appear to have developed chronic fibrotic lung disease.

1 Solé A, Marco V, Gonzalez A. A new pulmonary syndrome in workers of textile aerography (Ardystil syndrome). Immunological studies. Eur Respir f 1993;6:607s.

2 Moya C, Antó JM, Newman Taylor AJ, and the Collaborative Group for the Study of Toxicity in Textile Aerographic Group for the Study of Toxicity in Textile Aerographic

3 Colby TV. Pathologic aspects of bronchiolitis organizing pneumonia. Chest 1992;102:38-43s.

4 Epler GR. Bronchiolitis obliterans organizing pneumonia. Definition and clinical features. Chest 1992;102:2-6s.

5 Yamamoto $M$, Yasutaka I, Kitaichi $M$, Harasawa M, Tamura M. Clinical features of BOOP in Japan. Chest 1992;102: 21-25s.

6 Gregersen $P$. Neurotoxic effects of organic solvents in exposed workers: 2 . Controlled follow-up after $5 \cdot 5$ and $10 \cdot 6$ years. Am $\mathcal{F}$ Ind Med 1988;14:681-701. 